\title{
Elastic Wave Behaviour in 2-D Acoustic Interface Superlattices of an Altered Phase
}

\author{
Jerzy KAPELEWSKI, Bogdan LILA \\ Military University of Technology \\ Faculty of Electronics \\ Institute of Radioelectronics \\ Kaliskiego 2, 00-908 Warszawa 49, Poland \\ e-mail: jerzy.kapelewski@wat.edu.pl \\ (received November 10, 2010; accepted February 23, 2011)
}

\begin{abstract}
The purpose of the paper is to outline a systematic and unified non-local treatment of mode conversion effects associated with an interface superlattice being a 2D pseudo-array composed of altered phase inclusions (exemplified by impurity clusters) and located at a solid-solid plane interface. It will be illustrated, in some detail, for the instructive case of a Stoneley type acoustic wave (SW), incident on a periodically nonhomogeneous portion of an interface and partly transformed into bulk modes propagating in one of the component solid. An analytical model scheme is constructed, using a variational method combined with the $\mathbf{T}$ matrix approach, appropriate for the $2 \mathrm{D}$ periodic array treated, and decaying into the depth of this solid for the structure in a way determined by the array geometry and element 3D profiles as well as the boundary conditions at the interface. An apodization (weighting) to reduce the side-lobes level is incorporated into the structure by appropriately varying lateral dimensions and the depth of particular scatterers.
\end{abstract}

Keywords: acoustic antennas, elastic layers, interfaces.

\section{Introduction}

It is well known that a characterization of near-surface properties of materials by acoustic means is most conveniently done by surface acoustic waves (SAW), because they are very sensitive to surface inhomogeneities. In a series of previous works (KAPELEWSKI, PASTERNAK, 1996; 1999) we developed a method of using a surface 2D quasicomposite array composed of altered phase clusters which play the role of antenna elements when irradiating by a surface (Rayleigh) wave.

We have previously developed devices utilizing the new types of reflectors and interesting results have been obtained. We shall not dwell here on the merit of 
the method over more conventional electronically controlled 2D antenna arrays, which was thoroughly discussed elsewhere (KAPELEWSKI, PASTERNAK, 1996; 1999; KAPELEWSKI, 1991). In this paper we consider an analogous problem for the case when the array of altered phase clusters (as before (ANGEL, ACHENBACH, 1985; ClAUS, 1978) modelled by dipole-force dilation centers distribution in the host region) is situated at the interface between two (similar or identical but differently oriented) solids, and the excitation is provided by the SW propagating along the perturbed interface plane. The interface instead of surface has been used since the array plane has several new aspects. The interface structures of this kind can be implemented in various manners. It may be affected by interfering with the epitaxial growth process or by creating the interface through a mechanical adhesive contact of two samples, the one of which with a surface array treated previously. One important advantage of the interface localization of the $2 \mathrm{D}$ array in consideration, as compared to its surface version, lies in avoiding the contamination and another detrimental effect of the environment without recalling to high vacuum conditions. Generally the latter is rather necessary for the shortterm stability of working conditions for any phased array device of this kind.

Another aspect of the problem is treating it in terms of the non-destructive evaluations (NDE) of the inhomogeneous interface region, where the 2D periodic interface region can be viewed as a non-random periodic part of the local inhomogeneity profile of a material interface.

In case of the interface array, the exciting force for the antenna effect is provided by the SW which then play the role of SAW (Rayleigh wave) when going from the surface to the interface array systems of acoustical scatterers (reflecting elements). It has been long ago recognized that SW can be used very effectively to test the quality of solid-solid contacts, especially in detecting metal fatigue and testing the quality of adhesive bonds (ANGEL, ACHENBACH, 1985). The SW scattering in propagation through a nonhomogeneous interface should be the principal mechanism treated in such studies.

\section{Description of the model treatment}

An essential element of the method lies in taking advantage of an inherent structural analogy between analytic forms of the static Green's function for the two-component medium and that for typical acoustic waves of the Stoneley type given respectively by (DJAFARI-ROUHANI et al., 1977; 1980):

$$
\begin{array}{r}
\mathbf{G}\left(\mathbf{k}^{S} ; z, z^{\prime}\right)=\sum_{i, j}^{3} g^{i j}\left(\mathbf{k}^{S}\right) \exp \left(-q_{i} k^{S}|z|-q_{j} k^{S}\left|z^{\prime}\right|\right) \\
+\sum_{i}^{3} g^{i}\left(\mathbf{k}^{S}\right) \exp \left(-q_{i} k^{S}\left|z-z^{\prime}\right|\right)
\end{array}
$$


and

$$
\mathbf{u}_{0}^{(j)}\left(\mathbf{k}^{S}, z\right)=\sum_{n=1}^{3} \mathbf{u}_{0}^{n(j)}\left(\mathbf{k}^{S}\right) \exp \left[-q^{n(j)} k^{S}|z|\right], \quad j=1,2 .
$$

Following the model treatment, developed by us for a modified material surface (KAPELEWSKI, 1991) for the upper component (2) $(z>0)$, we obtain:

$$
\mathbf{T}^{S}\left(\mathbf{k}^{S}, z\right)=\sum c^{(i)}\left(\mathbf{k}^{S}\right) \exp \left(-\alpha_{i} z\right) \mathbf{P}^{(i)}+\mathbf{T}\left(\mathbf{k}^{S}\right) \exp (-\eta z)
$$

were $\eta$ is the decaying coefficient.

The SW scattered wave can than be written in the following form (KAPELEWSKI, 1991; Kapelewski, Pasternak, 1996):

$$
\begin{array}{r}
u_{\alpha}^{S}\left(\mathbf{k}^{S}, z\right)=\sum_{r s} g_{\alpha \beta, \gamma}^{r s}\left(\mathbf{k}^{S}\right) \exp \left(-q_{r} k^{S} z\right)\left[\sum_{i} \frac{c^{(i)}\left(\mathbf{k}^{S}\right) P_{\beta \gamma}^{i}}{q_{r} k^{S}+\alpha_{i}}+\frac{T_{\beta \gamma}\left(\mathbf{k}^{S}\right)}{q_{r} k^{S}+\eta}\right] \\
-\sum_{r} g_{\alpha \beta, \gamma}^{r}\left(\mathbf{k}^{S}\right)\left\{\sum_{i}\left[\frac{\exp \left(-\alpha_{i} z\right)-\exp \left(-q_{r} k^{S} z\right)}{q_{r} k^{S}-\alpha_{i}}+\frac{\exp \left(-\alpha_{i} z\right)}{q_{r} k^{S}+\alpha_{i}}\right] c^{(i)}\left(\mathbf{k}^{S}\right) P_{\beta \gamma}^{i}\right. \\
\left.-\left[\frac{\exp (-\eta z)-\exp \left(-q_{r} k^{S} z\right)}{q_{r} k^{S}-\alpha_{i}}+\frac{\exp (-\eta z)}{q_{r} k^{S}+\alpha_{i}}\right] T_{\beta \gamma}\left(\mathbf{k}^{S}\right)\right\} \\
+\sum_{r s}\left[g_{\alpha \beta}^{(r s)}\left(\mathbf{k}^{S}\right)+g_{\alpha \beta}^{(r)}\left(\mathbf{k}^{S}\right)\right]\left[\sum_{i} c^{(i)}\left(\mathbf{k}^{S}\right) P_{\beta z}^{i}+T_{\beta z}\left(\mathbf{k}^{S}\right)\right]
\end{array}
$$

with $\mathbf{T}^{S}$ denoting the interface internal stress, $\mathbf{P}^{i}$ and $\alpha_{i}$ describe, the dipole moments of the $i$-th kind dilation centers and the decaying of their concentration, respectively. The indices $r$ and $s$ denote the partial (longitudinal and shear) components of the SW, 1 and 2 is to number the component solids with the common interface.

The corresponding effective change in the elasticity tensor produced by the static bias, in the quasi-elastic approximation, can then be written in the form:

$$
H_{\alpha \beta}=T_{\alpha \gamma}^{S} \delta_{\beta \delta}+\delta C_{\lambda \beta \gamma \delta}^{S} \frac{\partial u_{\alpha}^{S}}{\partial x_{\lambda}}+\delta C_{\alpha \beta \lambda \gamma}^{S} \frac{\partial u_{\delta}^{S}}{\partial x_{\lambda}} .
$$

Then the variational formula for the relative full energy shift can be expressed by the relation

$$
\frac{\delta I\left(\mathbf{k}^{S}\right)}{P_{0}}=\frac{1}{2 \rho \omega^{2}} \frac{\int_{V}\left[S_{\beta \alpha} H_{\alpha \beta \gamma \delta} S_{\gamma \delta}^{*}-\delta \rho \omega^{2}\left|u_{\gamma}^{2}\right|\right] \mathrm{d} V}{\int_{V}\left|u_{\gamma}^{2}\right| \mathrm{d} V},
$$


where $\mathbf{S}(\mathbf{r}, z)$ is the dynamic strain tensor produced by the incident SW considered here to be an appropriate test function. The general form of the incident SW amplitude is given by:

$$
u_{\alpha}^{(j)}\left(\mathbf{k}^{S}, \mathbf{r}, t\right)=\sum_{n=1}^{3} \beta_{\alpha}^{n(j)} \exp \left[-q^{n(j)} k^{S}|z|+\mathrm{i}\left(\omega t-\mathbf{k}^{S} \cdot \mathbf{r}\right)\right],
$$

where $j=1,2$ denotes (as in (2)) solid components of the sample separated by the interface under consideration, $\beta_{\alpha}^{n(j)}$ are wave amplitudes of displacement vector components, $q^{n(j)}$ is the partial decaying coefficient; $\mathbf{k}^{S}$ - the SW wave vector; $\omega$ - the frequency; $|z|$ - the distance from the interface. The interface elastic energy density outgoing from the region considered, averaged over the period of time, can then be written:

$$
\delta I(\mathbf{r})=\frac{1}{4} \int_{0}^{\infty} D(\mathbf{r}, z) \mathrm{d} z-\frac{1}{4} \omega^{2} \int_{0}^{\infty} \delta \rho(\mathbf{r}, z)|u(\mathbf{r}, z)|^{2} \mathrm{~d} z \equiv \int I(\mathbf{r}, z) \mathrm{d} z,
$$

where $(\mathbf{r}, z)$ - the vector of position, $\mathbf{r}=(x, y), \delta \rho(\mathbf{r}, z)$ - the interface modification of the material density and $D=\mathbf{S} \mathbf{H} \mathbf{S}^{*}$ - an auxiliary quantity.

For the elastic stress tensor $\mathbf{T}(\mathbf{r}, z)$ in the quasilinear approximation, we have:

$$
\mathbf{T}(\mathbf{r}, z)=\mathbf{C}(\mathbf{r}, z) \mathbf{S}(\mathbf{r}, z)
$$

with $\mathbf{C}(\mathbf{r}, z)$ being the position depending elasticity tensor.

The Poynting formula for the c.w. case yields:

$$
\frac{1}{2} \operatorname{Re}\left[\mathrm{i} \omega \int_{S}-\mathbf{u}^{*} \cdot \mathbf{T} \cdot \widehat{\mathbf{n}} \mathrm{d} S\right]=\operatorname{Re}\left[\mathrm{i} \omega \int_{V} \delta I(\mathbf{r}, z) \mathrm{d} V\right] \equiv \int_{S} \mathbf{P} \widehat{\mathbf{n}} \mathrm{d} S,
$$

where: $\widehat{\mathbf{n}}$ - versor (unit vector) of the normal to the surface $S$ of the volume $V$; $\mathbf{P}$ - the Poynting vector; $\mathbf{u}$ - particle displacement. Having $\mathbf{P}$, we are able to calculate the attenuation of the propagating SW due to the coherent scattering to the bulk or other SW waves (i.e. those propagating in the directions other than the incident one).

Extending the approach for surface wave attenuation presented by Snieder (1988) to the case of interest here, we can evaluate the attenuation coefficient by means of the formula

$$
\gamma_{j}=\frac{4 N \sum_{i \neq j} P_{i}}{\omega\left(k^{S}\right)^{2}}
$$

with $P_{i}=i \omega J_{i}$ and $N$ being the number of scatterers for unit length along the SW propagation direction.

For simplicity of the description, we will concentrate themselves on the reasonable realistic case, when the modified interface is assumed to have an effective 
acoustic impedance just near to that of the one constituent solid (2), so that the scattering into the other solid constituent (1) is neglected (in the first approximation at least). Adopting this model assumption about the impedance matching, the dominant contribution to the integral in Eq. (10) is provided effectively by the clusters - solid 1 interface areas (denoted simply by $S_{a}$ hereafter).

For the periodically distributed pattern, the form factor of the structure is

$$
\Omega(\mathbf{k})=\sum_{\mathbf{l}} e^{i \mathbf{k} \mathbf{l}}=\frac{(2 \pi)^{2}}{S_{a}} \sum_{\mathbf{K}} \delta(\mathbf{k}-\mathbf{K}),
$$

where $\mathbf{K}=2 \pi \mathbf{h}$ with $\mathbf{h}$ being the points of the "reciprocate lattice" of the $2 \mathrm{D}$ cluster distribution.

In case of apodized 2D structure, the form factor can by written

$$
\Omega^{A}(\mathbf{k})=\sum_{\mathbf{l}} F(\mathbf{l}) e^{i \mathbf{k} \mathbf{l}}=\frac{(2 \pi)^{2}}{S_{a}} \sum_{\mathbf{K}} F(\mathbf{k}-\mathbf{K}),
$$

where $F(\mathbf{l})$ can be viewed as the apodization rule.

The respective variational formula for the scattering $\left(\mathbf{k}^{s} \rightarrow \mathbf{k}\right)$ coefficient can be presented in the following form (KAPELEWSKI, 2004)

$$
\begin{aligned}
R\left(\mathbf{k}, \mathbf{k}^{S}\right)=\operatorname{Re} \frac{i \omega}{P} \int\left[\mathbf{S}^{*}(\mathbf{k}, z) \delta \mathbf{H}\left(\mathbf{k}-\mathbf{k}^{S}, z\right) \mathbf{S}^{S}\left(\mathbf{k}^{s}, z\right)\right. \\
\left.-\delta \rho\left(\mathbf{k}-\mathbf{k}^{S}, z\right) \omega^{2} \mathbf{u}^{*}(\mathbf{k}, z) \mathbf{u}^{S}\left(\mathbf{k}^{S}, z\right)\right] \mathrm{d} z .
\end{aligned}
$$

For the first term in (14) we can write:

$$
\int \mathbf{S}_{0}^{*}(\mathbf{k}, z) \delta \mathbf{H}\left(\mathbf{k}-\mathbf{k}^{s}, z\right) \mathbf{S}_{0}^{S}\left(\mathbf{k}^{S}, z\right) \mathrm{d} z=\mathbf{S}_{0}^{*}(\mathbf{k}) \mathbf{H}_{0} \mathbf{S}_{0}^{S}\left(\mathbf{k}^{S}\right) \tilde{f}_{1}^{t}\left(\mathbf{k}-\mathbf{k}^{s}\right)
$$

with $\mathbf{S}_{0}, \mathbf{H}_{0}$ denoting, respectively, the amplitude of the strain and the value of $\mathbf{H}$ taken at $z=0$ is given by

$$
\widetilde{f}_{1}^{t}(\mathbf{k})=\int f(\mathbf{k}, z) \mathrm{d} z .
$$

The second term is then given in the same way (with $f_{1} \rightarrow f_{2}$ ) according to Eqs. (3)-(5). Denoting both $f_{1}$ and $f_{2}$ by $F$, we have then

$$
\widetilde{F}\left(\mathbf{k}-\mathbf{k}^{s}, z\right)=\sum_{\mathbf{l}} F(\mathbf{l}, z) e^{i\left(\mathbf{k}-\mathbf{k}^{s}\right) \cdot \mathbf{l}}=\sum_{\mathbf{K}} F\left(\mathbf{k}-\mathbf{k}^{s}-\mathbf{K}, z\right) .
$$

From the Poynting formula taken for all the structure, we then finally obtain:

$$
\delta I(\mathbf{k})=\frac{1}{4} \sum_{\mathbf{K}} \int\left[\mathbf{D}(K, z) f(\mathbf{k}-\mathbf{K}, z)-\omega^{2} \rho_{0}|\mathbf{u}(\mathbf{K}, z)|^{2} c(\mathbf{k}-\mathbf{K}, z)\right] \mathrm{d} z .
$$


The effective contribution to Eq. (18) is provided only by those components of $\mathbf{K}$ (i.e. for those "lobes") for which the dynamic matching conditions

$$
k_{\alpha}^{S}=k_{\alpha}-K_{\alpha}, \quad \alpha=x, y
$$

with

$$
k_{x}=k^{(m)} \sin \theta \cos \varphi, \quad k_{y}=k^{(m)} \sin \theta \sin \varphi
$$

are satisfied, and the superscript $(m)$ denotes the appropriate bulk or interface modes, $\theta$ and $\varphi$ are angles in the spherical coordinate system.

Let us notice that the scattering of the incident SW to an interface wave propagating in another direction (then expressed by $\varphi$ ), within its angular range of existence, is given directly by taking $\theta=\pi / 2 . \delta I(\mathbf{k})$ is the $2 \mathrm{D}$ Fourier transformed density of the elastic energy associated with the modified material interface given (according to Eqs. (8) and (10) by

$$
\delta I(\mathbf{r})=-\frac{1}{2} \int \mathbf{T}(\mathbf{r}, 0) \mathbf{u}^{*}(\mathbf{r}, 0) \cdot \widehat{\mathbf{n}} \mathrm{d} S
$$

In Eq. (18) $\mathbf{D}(k, z)=\mathbf{S C}_{0} \mathbf{S}^{*} f(k, z)$ and, in accordance with the model form given by Eq. (3), $\rho=\rho_{0} \sum c^{(i)}(\mathbf{k}) \exp \left(-\alpha_{i} z\right) \equiv \rho_{0} c(\mathbf{k}, z)$. The apodization (weighting) can be effected by controlling $c(\mathbf{k}, z)$.

Taking into account the foregoing effect of SW attenuation (for the case without apodization), Eq. (18) can be written:

$$
\begin{array}{r}
\delta I(\mathbf{k})=\frac{1}{4} \sum_{\mathbf{K}} \int \delta_{B}\left(\mathbf{k}^{\prime}-\mathbf{K}\right) \iint\left[\mathbf{S}(\mathbf{k}, z) \mathbf{C}_{0} \mathbf{S}^{*}\left(\mathbf{k}-\mathbf{k}^{\prime}, z\right) f\left(\mathbf{k}^{\prime}, z\right)-\right. \\
\left.-\omega^{2} \rho_{0} \mathbf{u}(\mathbf{k}, z) \mathbf{u}^{*}\left(\mathbf{k}-\mathbf{k}^{\prime}, z\right) c\left(\mathbf{k}^{\prime}, z\right)\right] \mathrm{d} \mathbf{k}^{\prime} \mathrm{d} z,
\end{array}
$$

where $\delta_{B}$ is the tempered $\delta$-function defined as

$$
\delta_{B}\left(k_{j}-K_{j}\right)=\frac{2 \gamma_{j}}{\gamma_{j}^{2}+\left(k_{j}-K_{j}\right)^{2}}, \quad j=x, y
$$

and $\gamma_{j}$ is the attenuation parameter along the SW propagation direction $j$.

In effect, Eq. (12) should be replaced by

$$
\Omega_{\mathrm{mod}}(\mathbf{k})=\sum_{l_{1}, l_{2}} e^{\mathrm{i} \mathbf{k} \mathbf{l}-\gamma_{1}\left|l_{1}\right|-\gamma_{2}\left|l_{2}\right|}=\frac{(2 \pi)^{2}}{S_{a}} \sum_{\mathbf{K}} \delta_{B}(\mathbf{k}-\mathbf{K}) .
$$

For the limiting case of $\gamma_{x}, \gamma_{y} \rightarrow 0$, we have $\delta_{B} \rightarrow \delta$, and (18) takes the "classical" form.

Having the array contribution to the interface and the dynamic stress tensor $\mathbf{T}(\mathbf{r})$, we are able to calculate the displacement field in the solid 2 by means of the Helmholtz-type formula, adapted in the Morse and FeshbaCH (1953) way 
to the solid support of the wave. For case considered here it can be rewritten in the form:

$$
\begin{aligned}
u_{\lambda}^{(2)}(\mathbf{r})=\int_{S} \sum_{\mathbf{L}}[ & -n_{\beta} T_{\alpha \beta}\left(\mathbf{r}^{\prime}\right) G_{\alpha \lambda}^{(2)}\left(\mathbf{r}-\mathbf{r}^{\prime}\right) \\
& \left.\quad+n_{\beta} \delta C_{\alpha \beta \gamma \delta}\left(\mathbf{r}^{\prime}\right) u_{\alpha}\left(\mathbf{r}^{\prime}\right) G_{\delta \lambda, \gamma}^{(2)}\left(\mathbf{r}-\mathbf{r}^{\prime}\right)\right] \delta\left(\mathbf{r}^{\prime}-\mathbf{L}\right) \mathrm{d} \mathbf{r}^{\prime}
\end{aligned}
$$

with

$$
T_{\alpha \beta}(\mathbf{r})=T_{\alpha \beta}(\mathbf{r}) e^{\mathrm{ikr}}, \quad u_{\alpha}(\mathbf{r})=u_{\alpha}^{0} e^{\mathrm{ikr}}
$$

and $\mathbf{n}$ denoting the direction normal to the surface, $\mathbf{G}^{(2)}$ is the part of the so called interface Green's function (IGF) corresponding to the specified component solid denoted here by 2 for the unperturbed two-solids system.

The 2D Fourier transformed IGF can be written in the form (KAPELEWSKI, PASTERNAK, 1996; 1999):

$$
\begin{aligned}
\widetilde{G}_{\alpha \beta}\left(\mathbf{k}, z, z^{\prime}\right)=\sum_{k=1,2,3} \delta_{\alpha \beta} g(1)_{\beta}^{k}(\mathbf{k}) e^{-\alpha_{k}\left|z-z^{\prime}\right|} & +\sum_{\substack{k=1,2,3 \\
l=1,2,3(1) \\
1^{\prime}, 2^{\prime}, 3^{\prime}(2)}} g(2)_{\alpha \beta}^{k l}(\mathbf{k}) e^{-\alpha_{k}|z|+\alpha_{l}\left|z^{\prime}\right|}
\end{aligned}
$$

where $\alpha_{k}=\sqrt{k^{2}-\omega^{2} / v_{k}^{2}}, k$ is the number of the bulk modes involved.

Taking the 2D Fourier transform of Eq. (25), we arrive at the formula

$$
\begin{aligned}
u_{\lambda}^{(2)}(\mathbf{k}, z)=\sum_{\mathbf{K}} \int n_{\beta}[ & -G_{\alpha \lambda}^{(2)}\left(\mathbf{k} ; z, z^{\prime}\right) T_{\alpha \beta}^{0}(\mathbf{k}-\mathbf{K}) \\
& \left.+u_{\alpha}^{0}(\mathbf{k}-\mathbf{K}) \delta C_{\alpha \beta \gamma \delta}(\mathbf{k}) G_{\gamma \lambda, \delta}^{(2)}\left(\mathbf{K} ; z, z^{\prime}\right)\right] \mathrm{d} z^{\prime}
\end{aligned}
$$

determining the coherently scattered wave. The angular dependence of Eq. (15) expresses the respective directivity patterns for the Fourier transformed Poynting vector $\mathbf{P}(\mathbf{k})$ (see Eq. (10)).

Results of exemplary numerical simulations, realized for chosen pairs of isotropic materials, are pictured in Fig. 1. For both the used materials, the Weichert's criteria (CLAUS, 1978) $\frac{\lambda_{1}^{L}}{\lambda_{2}^{L}}=\frac{\mu_{1}^{L}}{\mu_{2}^{L}}=\frac{\rho_{1}}{\rho_{2}}\left(\lambda^{L}, \mu_{1}^{L}\right.$ - the Lame constants), have beeen assumed. 

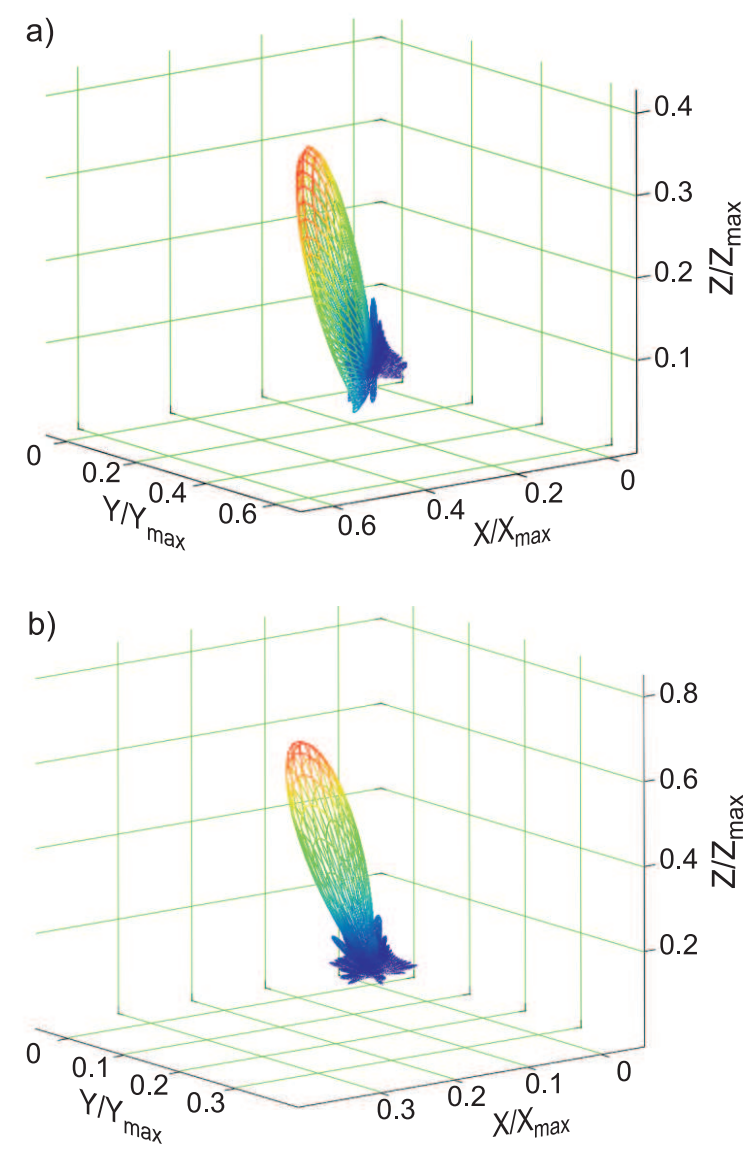

Fig. 1. Exemplary normalized profile of power conversion ratio for shear (a) and longitudinal (b) bulk waves. Cluster-solid 2 interfaces are of the circular shape $(r=0.25 \lambda)$. The Gaussian depth profile $\left(z_{n}=0.2 \lambda, d_{n}=0.3 \lambda\right), \mu_{1} / \mu_{2}=0.339, \rho_{1} / \rho_{2}=0.352$. In the cluster area $\mu_{3}=1.08 \mu_{1}$ and $\rho_{3}=1.1 \rho_{1}$.

\section{Conclusions}

The approach presented deals with a specific physical situation when the 2D phased array plays a role of a kind of an acoustic interface antenna for the anticipated NDE application. The array is assumed to be composed of clusters having material parameters slightly different to those of the host which can be both abrupt and slowly varying, even within particular clusters areas. Given the impurity kind and concentration of the dilation of the perturbed, specifically varying, elastic parameters can essentially be determined from the static equilibrium equation, which we expressed indirectly by means of the 2D Green's function in terms of the so called interface fluctuation waves. Being especially well fitted to the dipole-force model of the impurity distribution, it makes the problem tractable in analytical terms. 
For simplicity, both the solids have been assumed in the tentative numerical simulation (Fig. 1) to be isotropic. The incident interface wave itself was considered to be excited externally (i.e. without using interdigital transducers), so the substrate solid itself need not to be piezoelectric.

The proposed treatment seems to be useful for modelling and optimization of the bulk waves scattered by various arrays of interface clusters of the type considered here. The approach can readily be extended to describe 3D focussing and beam-forming of the bulk waves coherently scattered into the depth by the mechanism under consideration (both are then governed by the frequency modulation of the incident SW) for the anticipated use in NDE of the bulk material used. The treatment of some related problems are now being under way.

\section{References}

1. Angel Y.C., Achenbach J.D. (1985), Reflection and Transmission of Elastic Waves by a Periodic Array of Cracks, Journal of Applied Mechanics, 52, 33-41.

2. Claus R.O. (1978), Effects surface bonding on the existence of ultrasonic Stoneley waves, Proceedings of the IEEE Ultrasonics Symposium, pp. 396-399.

3. Djafari-Rouhani B., Dobrzyński L., Maradudin A.A., Wallis R.F. (1980), Elastic interactions between defects and interfaces, Surface Science, 91, 618-628.

4. Djafari-Rouhani B., Dobrzyński L., Wallis R.F. (1977), Elastic continuum theory of interface-atom mean-square displacements, Physical Review B, 16, 741-749.

5. Kapelewski J. (2004), Coherent Scattering and Acoustic Wave Conversion in Monolithic Interface 2D Arrays, Proceedings of the 11th Annual International Conference on Composites/Nanoengineering (ICCE - 11), Hilton-Head, USA.

6. Kapelewski J., Pasternak M. (1999), A systematic approach to near surface distortion produced by paraelastic impurity systems, Biuletyn WAT, 48, 6, 113-123.

7. Kapelewski J., PAsternak M. (1996), Surface acoustic waves in quasi-composite nearsurface microstructured layers, Composite Interfaces, 3, 425-430 .

8. KAPELEWSKI J. (1991), A near surface defect-induced lattice distortion, Proceedings of the 5th International, Symposium on Nondestructive Characterization of Materials, pp. 533539, Karuizawa, Japan.

9. Morse P., Feshbach H. (1953), Methods of Theoretical Physics, McGraw-Hill, New York.

10. SNiEder R. (1988), The optical theorem for surface waves and the relation with surfacewave attenuation, Geophysical Journal, 95, 293-302. 